\title{
A comparison of different techniques for atmospheric artefact compensation in GBSAR differential acquisitions
}

\author{
Luca Pipia, Xavier Fabregas, Albert Aguasca, Jordi. J. Mallorqui \\ Universitat Politecnica de Catalunya (UPC), \\ D3-Campus Nord-UPC, C/ Jordi Girona 1-3, 08034 Barcelona, Spain \\ \{luca.pipia, fabregas, aguasca , mallorqui\}@tsc.upc.edu
}

\begin{abstract}
In this paper, a comparison of different techniques for compensating the atmospheric artifacts in Ground-Based SAR zero-baseline acquisitions at $\mathrm{X}$-Band is presented. The way the fluctuation of atmospheric parameters like temperature, pressure and humidity among successive scans affects interferometric phases is pointed out and a simple model based on the refractivity index variations is given to explain such a behavior. Three alternative methods for the artifact removal, respectively based on the differential phase 1D unwrapping, 2D unwrapping and the Chirp-Z transformation are described and quantitatively compared using a reference dataset. The advantages and the limitations of each technique are finally drawn.
\end{abstract}

Keywords- Ground-Based Sensor, Refractive Index, In-SAR and DInSAR.

\section{INTRODUCTION}

The high stability of the sensor platform and the lack of revisiting-time constrains, which usually limits any monitoring activity, constitute the most relevant advantages that the usage of Ground-Based SAR systems guarantees. In the latest years several efforts have been made for improving the performance these microwave sensors that are imposing as a promising alternative to the satellite solution when small scenarios are dealt with. An X-Band PolInSAR system has been developed at the Universitat Politecnica de Catalunya (UPC) [1]. A first differential PolSAR measurements campaign carried out in Collserola, close to the city Barcelona has pointed out the deep influence of atmosphere on interferometric phase even in case of short range distance and short time separations among acquisitions [2]. In this paper a set of compensation techniques for atmosphere effects removal in GB differential acquisitions is proposed. In Section II the UPC sensor is briefly described and the main steps of the focusing process are summarized. In Section III the relation between the fluctuation of the atmospheric parameters as temperature, pressure and humidity and the differential phase is explicited using a linear model. An observation period of one week is taken into account to stress the way the interferometric phase is corrupted. In Section IV three alternative methods for the atmospheric differential phase artifact removal are analyzed. Two of them are based on the determination of the regression line that best fit the inteferometric phase distribution of the high coherence pixels by a $1 \mathrm{D}$ and $2 \mathrm{D}$ phase unwrapping approach, respectively. The third method deals with a spectral analysis of a high coherence area using the Chirp-Z transformation. In order to assess the performance of the difference techniques a displacement has been simulated within the scenario. A Polarimetric Active Radar Calibrator (PARC) has been placed upon a micrometric positioner and displaced according to defined sequence of movements. In Section $\mathrm{V}$ the results obtained by applying the compensation methods in order to retrieve the displacement information are presented. The advantages and drawbacks of the different techniques are finally discussed and a general criterion for the selection of a method depending of the features of the observed area is provided.

\section{UPC GBSAR SENSOR}

The UPC GB sensor constitutes the result of an engineering project that aimed at optimizing the performance of a SAR ground platform in any acquisition configuration, from the simplest single-polarization to the most troublesome PolInSAR mode, at minimizing the sensor weight and size for a higher portability, and at reducing costs. Respect to the most of GB systems available in the remote sensing scientific community, which backs up on a Vector Network Analyzer (VNA) for the stepped-frequency sweeping of the transmitted signal bandwidth [3][4], the UPC CW-FM radar is based on a Digital Direct Synthetizer (DDS) chipset which generates it ance. This solution allows to sensitively reduce the time the sensor has to stop at each position of the synthetic aperture and provides the opportunity to perform PolSAR or PolInSAR measurements without increasing the temporal decorrelation effects during a single scan. The system works at X-Band although its modular structure allows to change the working frequency carrier with few hardware modifications involving the antennas block and the DDS parameters set-up. The processing algorithm is divided into two main steps: range compression and azimuth focusing. The GB sensors using a VNA perform the range compression by an IFFT transformation of echoes collected at different frequency carriers; the UPC GB-SAR acquires the raw data directly in the temporal domain. For this reason, after being averaged at hardware level and deramped, the signal is range-compressed by performing a simple FFT. Owing to the particular geometry of the system and to the fact the cross-range resolution is not a constant parameter in the image, back-projection revealed to be the most suitable technique for the azimuth focusing step [5]. A 
hamming filtering is also applied both in range and azimuth focusing steps in order to reduce the level of side lobes.

\section{ATOMOSPHERIC ARTEFACT}

A well-known empirical relation between the parameters of temperature $T$, pressure $p$ and humidity $h$ and refractive index $n$ is [6]:

$$
n=1+\left(77.6 \frac{p}{T}+600 \frac{p}{T^{2}} h \cdot 10^{12.22-\log _{10}(p)-\frac{2360}{T}}\right)
$$

The absolute phase of the EM monochromatic wave at frequency $f_{\mathrm{c}}$ backscattered from a point target $\mathrm{P}$, which is placed at a range distance $r_{n}$ from the radar sensor, can be expressed as:

$$
\varphi=4 \pi f_{c} t_{n}=4 \pi f_{c} \frac{r_{n}}{c} \iint_{t} d n=4 \pi f_{c} \frac{r_{n}}{c} n
$$

where $c$ is the speed of light. In equation (2) it is also emphasized that $n$ is generally a time-space function and that the final absolute phase is the result of the fluctuation of $n$ during the two-way propagation through the medium. Under the hypothesis of temporal stationarity and spatial homogeneity for the atmosphere, the index $n$ can be supposed to be just a function of time. Moreover, the temporal variations are usually slow enough to take $n$ constant during one measurement, as stressed in the last equality of eq.(2). If now the echo from a motionless point target $\mathrm{P}$ is acquired under slightly different weather conditions in a perfect zerobaseline configuration, its differential phase is not null because a phase variation occurs:

$$
\Delta \varphi=4 \pi f_{c}\left(t_{n 2}-t_{n 1}\right)=4 \pi f_{c} \frac{r_{n}}{c}\left(n_{2}\left(t_{2}\right)-n_{1}\left(t_{1}\right)\right)
$$

It can be noticed that $\Delta \varphi$ is proportional to the carrier frequency: at X-Band this effect is expected to be stronger than at lower bands like $C$ or $L$. Besides, this quantity is a function of the target position itself: if the point $\mathrm{P}$ moves on a $2 \mathrm{D}$ plane, the term $\Delta \varphi$ defines a linear phase ramp proportional to the target range-distance. In order to study this phenomenon, a collection of one week dataset acquired in October 2005 of the test area of Collserola hill (figure 1), close to the City of Barcelona has been used. In figures 2a-b the HH differential coherence and phase corresponding to two zero-baseline dataset acquired with 5 hours delay are displayed, respectively. The Figure $2 \mathrm{~b}$ clearly provides an example of the theoretical atmospheric artifact described by eq.(3) and confirms the correctness of the model. The differential phase is clearly wrapped although the short time separation. The millimetric displacement of a high coherent target placed about $600 \mathrm{~m}$ far from the sensor related to temporal evolution of the polarimetric differential phase during the observation period is plotted in Figure 3. The interval time between successive acquisitions is approximately 1 hour. The large fluctuations of interferometric phase, which affect the same way the different polarimetric channels, prevent from profitably using the collected data if not properly removed.

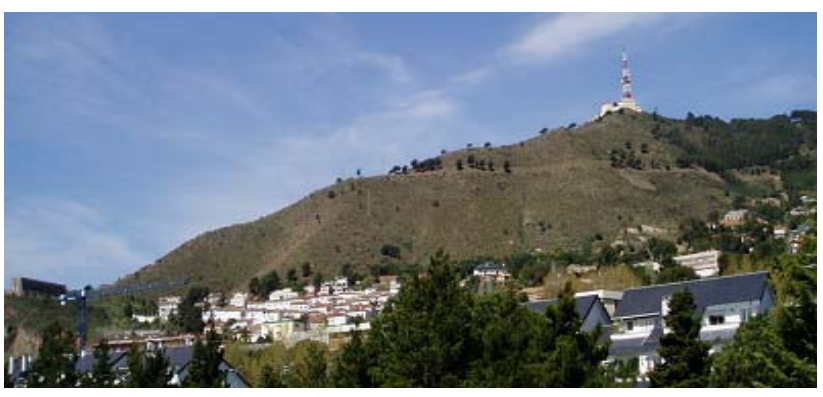

Figure 1: Collserola hill test-area

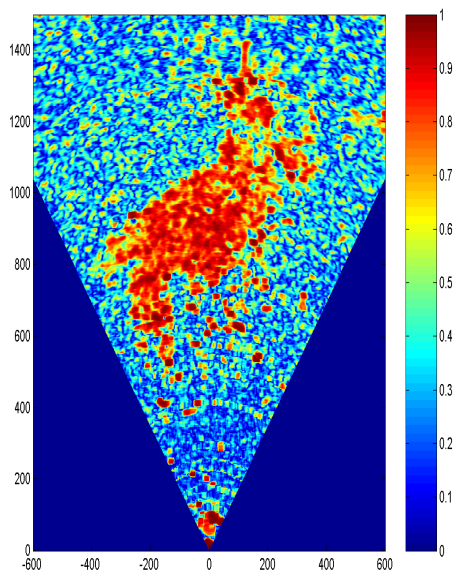

(a)

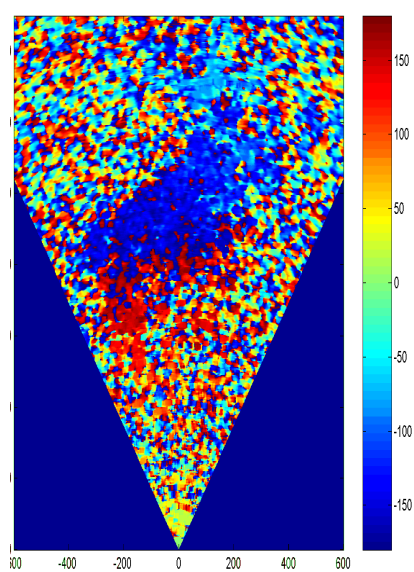

(b)
Figure 2: $5 \mathrm{~h}$ delayed zero-baseline HH diff. coherente (a) and phase (b)

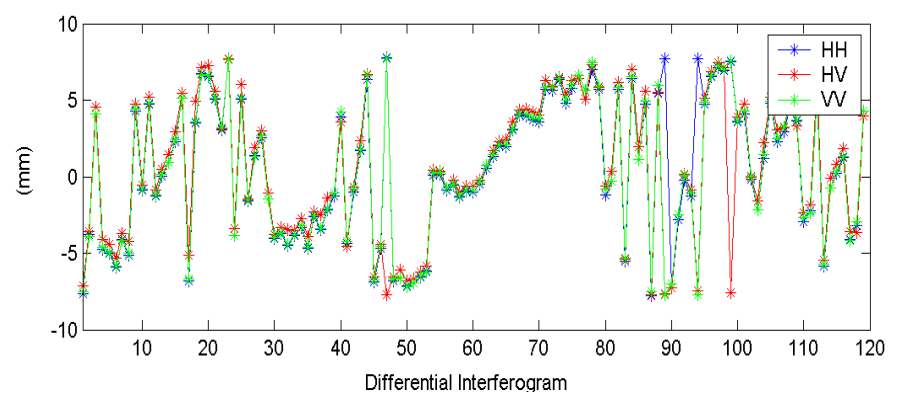

Figure 3: Apparent Displacement (mm) during 1 week

\section{ATOMOSPHERIC ARTIFACT REMOVAL}

In order to apply any differential interferometric technique for deformation map retrieval, the spurious effects introduced by atmosphere must be compensated for. The linearity that the phenomenon described in the previous section shows in most of cases suggests looking for compensation method based on the estimation of the surface best-fitting the phase ramp. Motionless high-coherent pixels are selected as interferogram reliable points. Since phase usually wraps, an unwrapping algorithm must be applied: the 2D and 1D solutions have been looked into.

It is worth of interest to remind that the points at the same range distance are affected the same way by atmosphere. Respect to satellite or airborne SAR data, where the range distance can be identified with one of the two focused image dimensions, in the GB SAR data it corresponds to the radial 
distance of each point within the image respect to the centre of the synthetic aperture reference. Consequently, under assumption of atmosphere spatial homogeneity, the artifact consists of a linear range phase-ramp that can be theoretically described by just two parameters: the offset and the angular coefficient. The projection onto a range-line of the phase of all the pixels whose coherence value is higher than a reference value is expected to show a linear behavior. A simple regression-line estimator can be hence used to find the ramp angular coefficient and the offset.

There are two possible alternatives to exploit this geometrical feature of the GBSAR data. Differential phase can be first unwrapped using a $2 \mathrm{D}$ phase unwrapping technique so that the phase lines up directly after the range projection. The other solution defines as first step the projection and then the differential phase unwrapping using a simpler 1D algorithm.

In Figure 4 an example of the compensation ramp estimation obtained in the two cases is displayed. The orange points correspond to the phase distribution obtained from the first solution (an algorithm based on a combination of the WLMS and the region-growing methods has been used), the green and blue points from the second technique, before and after 1D unwrapping step respectively. The results are very similar: the offset of the 2D unwrapping approach arises from the numerical algorithm and it is easily removable since the regression line is expected to start in the reference origin. The numerical value of the angular coefficients is almost the same although in the $2 \mathrm{D}$ unwrapping phase it can be noticed that a group of high coherent targets about $400 \mathrm{~m}$ far (blue circle) goes away from the linear trend and makes the two values differ. The hypothesis of atmosphere homogeneity is fulfilled for the two dataset taken into account so that the shift is a consequence of a local offset of the 2D routine that does not appear in the 1D solution (blue-dotted circle). The reason lies in the fact that, even after few hours delay, the X-Band differential coherence of distributed targets appreciably decreases and the interferogram obtained by filtering out the low coherence pixels, depending on the features of the observed scenario, might reduce to a sparse matrix. Sparse matrices represent the most troublesome problem any 2D unwrapping algorithm is to deal with and might lead to incorrect results. This problem does not occur in the $1 \mathrm{D}$ approach since the projection of the whole information onto a unidimensional space allows to fill up the potential empty areas that the 2D approach or a single range cut could present. Moreover, the time required for linear compensation parameters estimation is shorter. Nevertheless, depending on the observed scenario, the atmosphere could not behave uniformly over the whole image and a slightly different compensation might be required for different angular sectors.

The third method proposed in this paper for the atmospheric artifact removal is based on a completely different idea. From the spectral point of view, the variation of the propagation refractive index introduces a tone in the interferogram bidimensional spectrum. The spectral component of this almost monochromatic signal, like the angular coefficient estimated with the first two methods, is very low. Owing to

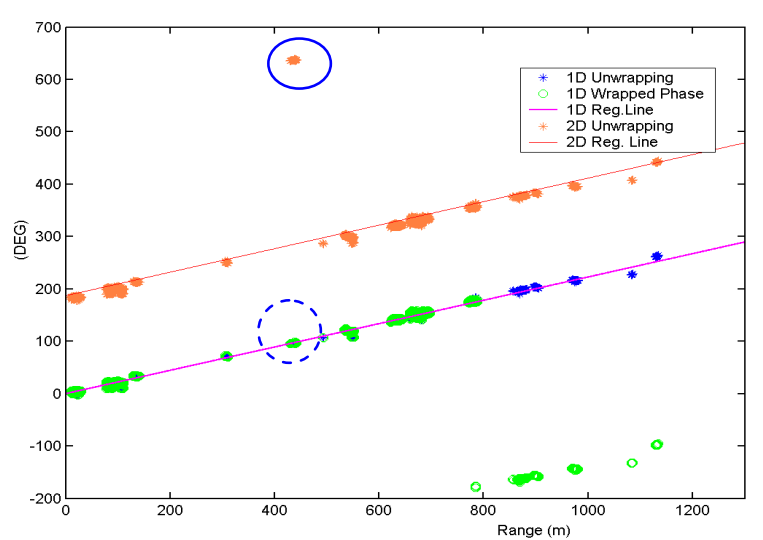

Figure 4: $1 \mathrm{D} / 2 \mathrm{D}$ phase unwrapping - Regression Line Estimation

the spatial sampling step of the GBSAR focused image $(1.5 \mathrm{~m} \times 1.5 \mathrm{~m})$ and the maximum range the sensor can observe, it is not possible to correctly estimate the value of this atmospheric carrier by means of a simple FFT analysis, neither if a high interpolation factor is used. The inherent limitation of the FFT algorithm, which provides a description of the whole spectral range defined by the sampling step but does not allow to focus just on a frequency axis interval of interest, prevent from obtaining a good estimation. A wellknown mathematical tool for short interval frequency analysis is the bidimensional Chirp- $Z$ transform (CZT), often used for the study of digital filter responses. Since just very low frequency components can be caused by the atmospheric changes - their correlation length within the image is very high - the Chirp-Z technique allows to overcome the constraints of the classical spectral analysis. If this spectral approach is used, the unwrapping step is unnecessary and the technique provides a high precision in the atmospheric carrier estimation. The main drawback is that it requires a sufficiently wide high-coherence homogeneous motionless area where to apply the transform: depending on the scenario and the time delay between acquisitions, finding such a kind of zone might reveal troublesome. It is worthy to underline that due to the radial symmetry of the atmospheric phase ramp, the $2 \mathrm{D}$ spatial carrier cannot be correctly estimated using a Cartesian referenced image: the spectral analysis of the differential phase in the xy axis provides a description of the spatial frequency as a superposition of kx-ky planes, which cannot approximate the atmospheric radial function:

$$
e^{j k_{a t m} \sqrt{x^{2}+y^{2}}}=e^{j k_{a t m} r} \neq e^{j \hat{k}_{x} x+j \hat{k}_{y} y}
$$

Equation (4) shows that GBSAR data must be processed in polar coordinates in the CZT technique for useful results. The results of 1D regression-line technique and the CZT method concerning a motionless corner reflector placed within the scene is given in figures 5 and 6 , respectively.

\section{COMPENSATION TECHNIQUES ASSESSMENT}

In order to assess the performance of the proposed compensation coherence-based techniques, a target displacement has been simulated using a reference point. A 


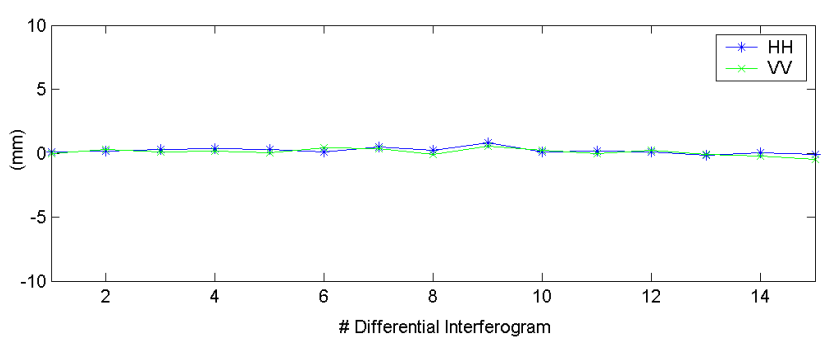

Figure 5: CR differential phase - 1D Reg. Line Compensation

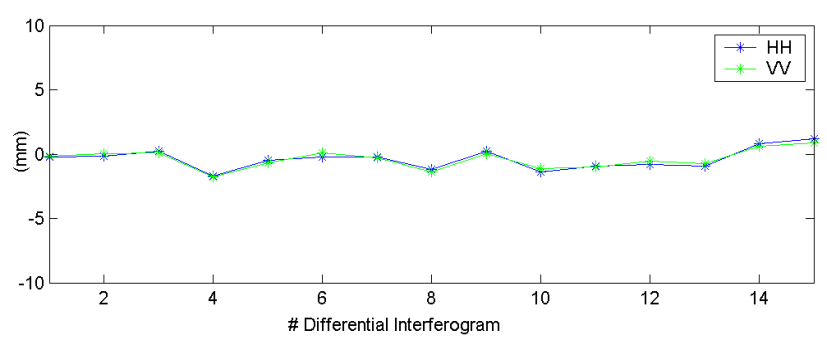

Figure 6: CR differential phase - CZT Compensation

HV PARC (Polarimetric Active Radar Calibrator) has been placed within the scenario about $1 \mathrm{~km}$ far from the sensor, where atmosphere dramatically corrupts any differential phase information, and $45^{\circ}$ tilted to furnish a backscattering signal in the four polarimetric channels. The corresponding $[\mathrm{S}]$ matrix is:

$$
\left[S_{P V H}^{45^{\circ}}\right]=\frac{S_{d}}{2}\left[\begin{array}{cc}
-1 & -1 \\
1 & 1
\end{array}\right]
$$

The device has been mounted on a micrometric positioner and its position has been modified between two successive acquisitions, according to the predefined sequence. Although the three techniques described in Section III have been used, the $1 \mathrm{D}$ and $2 \mathrm{D}$ techniques provide very close results and only the first one is here reported. The retrieved displacements sequence in the different polarimetric channels after the atmospheric artifact removal in the 1D Regression Line and the CZT cases is compared to the real one in figures 7 and 8 . A good agreement is detectable in both cases. Nevertheless, the spectral technique turns out to be less reliable than the 1D method, as clearly shown by both the trihedral and the PARC displacement profiles: sudden fluctuations from the real position occur in the CZT retrieval, leading to a not correct estimation of target position. This result, which apparently points out a higher reliability of the first method, has not to be related to a theoretical limitation of CZT technique but to the dominant features of the observed scene: the homogeneous high-coherent area that CZT requires had to be selected in the low/medium vegetated area of the hill slope, where atmosphere instability increases the signal correlation and reduces the interferometric coherence. Owing to the small scenario the GBSAR sensor is able to observe, no other part of the scenario can be used for such a purpose. From this point of view, the 1D method overcomes this limitation because it takes profit of all the high-coherent pixels inside the image, independently of their positions. In conclusion, although the three methods are theoretically equivalent, they are based on

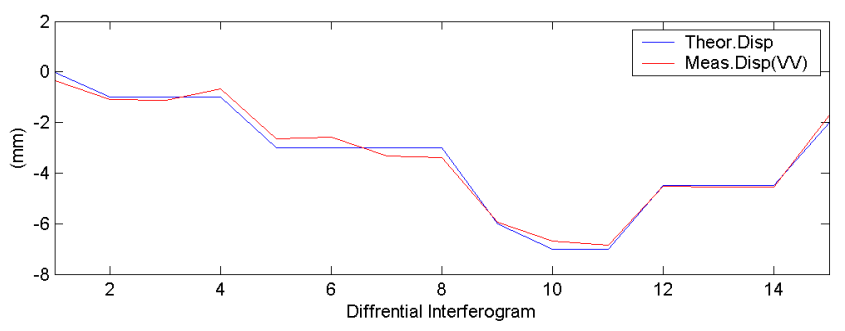

Figure7: Parc Displacement Retrieval: 1D Reg. Line

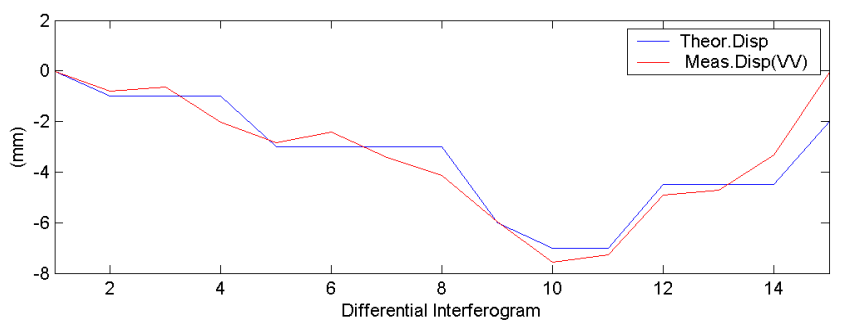

Figure8: Parc Displacement Retrieval: CZT Method

different scenario hypothesis of coherence and homogeneity: for this reason the best compensation is achieved using the method that better matches the scenario features.

\section{CONCLUSIONS}

In this paper the potential of GBSAR sensor for differential applications has been pointed out. Despite several advantages related to the high stability this sensor platform guarantees, the atmosphere instability turns out to deeply corrupt interferometric information at X-Band. Three alternative techniques for the phase artifacts removal have been proposed and assessed using active and passive reference points within the scenario. Finally, a general criterion for the selection of the compensation method has been given.

\section{ACKNOWLEDGMENT}

This work has been funded by the Spanish MCYT under the project TEC2005-06863-C02-01.

\section{REFERENCES}

[1] Aguasca et al.," A Solid State L to X-band Flexible Ground-based SAR System for Continuous Monitoring Applications", IGARSS 2004, Anchorage, Alaska

[2] L.Pipia et. al, "Temporal Decorrelation in Polarimetric Differential Interferometry using a Ground-Based SAR Sensor”, IGARSS'05, Seul, Korea, 25-29 July 2005

[3] Z.Zhou et al., "Development of a Ground-Based Polarimetric Broadband SAR System for Noninvasive Ground-Truth Validation in Vegetation Monitoring," IEEE TGRS, Vol. 42, No. 9, September 2004

[4] Nico,D.Leva et al.,"Generation of Digital Terrain Models With a Ground-Based SAR System," IEEE TGRS, Vol. 43, No. 1, January 2005

[5] M.Soumekh, "Synthetic Aperture Radar Signal Processing", Wiley \& Sons Inc., New York,1999

[6] Lucien Boithias, "Propagationdes ondes radioélectriques dans l'environnement terrestre," ordas et CNET-ENST,Paris, 1984.city and Magnetism, 3rd ed., vol. 2. Oxford: Clarendon, 1892, pp.68-73. 